\title{
Versorgungsforschung ist Dienst an der Bevölkerungsgesundheit
}

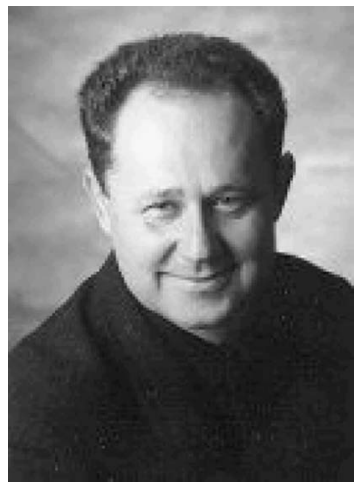

Prof. Dr. med. Manfred Wildner

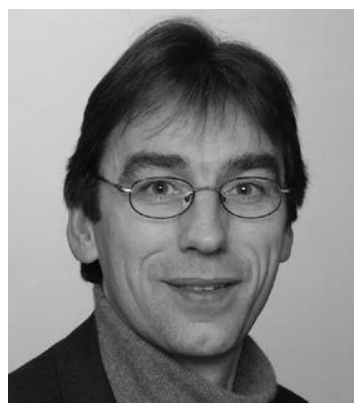

PD Dr. Alfons Hollederer
Bibliografie

DOI http://dx.doi.org/

$10.1055 / \mathrm{s}-0035-1547267$

Gesundheitswesen 2015;

77: 131-132

(c) Georg Thieme Verlag KG

Stuttgart · New York

ISSN 0941-3790

Korrespondenzadresse

Prof. Dr. med. Manfred Wildner

Bayerisches Landesamt für

Gesundheit und Lebensmittel-

sicherheit (LGL)

Veterinärstraße 2

85764 Oberschleißheim

manfred.wildner@|gl.bayern.de
Es ist eine große Freude, das vorliegende Heft zum Schwerpunkt Versorgungsforschung mit einem Editorial zu begleiten. Einerseits deswegen, weil unsere Zeitschrift organschaftlich mit dem Deutschen Netzwerk Versorgungsforschung (DNVF) verbunden ist. Andererseits begründet sich die Freude auch in der Sache selbst. Die vorliegenden Beiträge zur Versorgungsforschung markieren einen beachtenswerten Schritt kluger (Um-)Orientierung, welcher auf der Bundesebene und in unterschiedlichem Maß auch in verschiedenen Bundesländern getan worden ist und getan wird. Diese Aktivitäten sind notwendige und von unterschiedlichsten Seiten lange erwartete Schritte in eine Zukunft der gesundheitlichen Versorgung, welche vermehrt durch transparente Diskussionen und gemeinsame Reflexionen von gesundheits- und versorgungsbezogener Evidenz geprägt ist.

Die in der vorliegenden Schwerpunktausgabe enthaltenen Beiträge entstanden im Rahmen einer Initiative zur Versorgungsforschung eines Bundeslands, Bayern, und mit Unterstützung des Bayerischen Ministeriums für Gesundheit und Pflege. Für die Zeitschrift „Das Gesundheitswesen“ sind Schwerpunktausgaben ein willkommener Akzent, der Mehrumfang ein Bonus für Sie als Leser. Nur als Beispiele sei auch auf die Aktivitäten zur Versorgungsforschung in anderen Ländern wie Nordrhein-Westfalen (www.lzg.gc.nrw.de) und Baden-Württemberg (www.versorgungsfor schung-bw.de) hinwiesen.

International hat die Versorgungsforschung unter dem Begriff des Health Services Research ein breites Spektrum an Themen. Diese fanden insbesondere in den angloamerikanischen und skandinavischen Ländern deutlich früher eine breite und offene Rezeption als im deutschen Sprachraum. Nicht nur verschiedene patientenzentrierte Behandlungsergebnisse („Outcomes“) stehen hier im Fokus - erwünschte wie unerwünschte -, sondern mit ihnen auch die Strukturen und Prozesse, die zu Ihnen geführt haben $[1,2]$. Auch wenn international in der Historie gern auf die klassischen Forschungsarbeiten aus den Neuenglandstaaten der USA hingewiesen wird, wie jene von John Wennberg $[3,4]$, führen die Traditionslinien doch auch nach Deutschland und insbesondere zu Manfred Pflanz nach Hannover, welchen Wennberg persönlich besucht hatte [5].

Versorgungsforschung ist im 21. Jahrhundert nun auch in Deutschland wieder ein zentrales Thema geworden - hier verbindet sich also Neues wieder mit Altem. Dass das so erzeugte Wissen einen wichtigen Beitrag zur Bewusstseinsbildung und Entscheidungsunterstützung nicht nur auf Bundesebene, sondern auch auf Landesebene leisten kann, belegen die hier vorliegenden Beiträge. Den Auftakt bildet ein Memorandum, das die bayerische Landesarbeitsgemeinschaft Gesundheitsversorgungsforschung (LAGeV) erstellte, um auf Basis einer gemeinsamen Bedarfsanalyse grundlegende Zukunftsthemen und Entwicklungspotenziale für die eigenen Strategien und Maßnahmen zu identifizieren und festzuhalten (Hollederer et. al.). Es schließt an die bewährte Tradition in der Versorgungsforschung an, „Denkund Erinnerungswürdiges“ als Memorandum zu bewahren. Dem Memorandum ging eine strukturierte Befragung zum Stand und zu Potenzialen der Versorgungsforschung voraus, die ebenfalls in der Schwerpunktausgabe in einem Artikel von Hollederer, Voigtländer, Wildner, Zapf und Zellner veröffentlicht wird. Die Ergebnisse der Befragung und die Schwerpunkte im Memorandum folgen einerseits der regionalen Perspektive der Akteure, sind aber andererseits weitgehend anschlussfähig an die nationalen Entwicklungen zur Versorgungsforschung und lassen sich größtenteils auf andere Regionen übertragen. Möglicherweise stellt der Ansatz, Versorgungsforschung in Form einer Landesarbeitsgemeinschaft nach dem „Bottom up-Prinzip“ regional zu stärken, auch für weitere Bundesländer eine realistische Handlungsoption dar.

Die vorliegende Schwerpunktausgabe Versorgungsforschung umfasst darüber hinaus Grundlagenartikel und Versorgungsforschungsstudien. Donath, Gräßel et al. fragen in ihrem Beitrag nach den Grundsätzen der Evidenzbasierung in der Versorgungsforschung und beschreiben einen Kreislauf evidenzbasierter Versorgungsforschung im Kontext medizinischer Forschung. Im Mittelpunkt des Gesundheitswesens steht der Mensch, im Krankheitsfall als Patient. Orientiert sich aber die Versorgungsforschung am Patienten? Und wenn ja, auf welche Art und Weise? $\mathrm{Zu}$ diesen zentralen Fragestellungen geben Apfelbacher et al. einen Überblick. Braun und Binder beleuchten dagegen überblicksmäßig Vergütungsstrukturen von Gesundheitswerken. A. Schneider führt eine Bestandsanalyse der Versorgungsforschung in der Allgemeinmedizin durch. W. Schneider, Eichner, Thoms, Stadelbacher und Kopitzsch eruieren des Weiteren die Wirksamkeit und Effekte der Spezialisierten Ambulanten Palliativversorgung (SAP) aus sozialwissenschaftlicher Perspektive. Clarner, Krahl, Uter, Drexler und Martin untersuchen in einer Pilotstudie die psychische Traumatisierung 
nach Arbeitsunfällen bei Fahrdienstmitarbeitern und ihre Einflussfaktoren. Speziell für den Öffentlichen Gesundheitsdienst wird ein adäquates theoretisches Modell zur Versorgungsforschung im Beitrag von Hollederer und Wildner vorgeschlagen. Die Gesamtschau der Artikel in dieser Ausgabe zeigt auch auf, dass sich die Versorgungsforschung in Deutschland mittlerweile in vielen Themenfeldern etabliert hat, dabei insgesamt noch stark ausbaufähig ist und großes Potenzial in den und für die Regionen hat.

Weitere Beiträge in diesem Heft beschäftigen sich mit Sozialmedizin und normativer Versorgungsforschung (SalomonNeumann-Festvortrag), der Frage nach einer Zunahme gesundheitlicher Ungleichheit, sedentärem Verhalten und Gesundheit, Gesundheitskonferenzen in Deutschland, ambulant-sensitiven Krankenhausfällen und dem Spannungsfeld von Ressourcenallokation und persönlicher Autonomie.

Erhebliches Potenzial hat Versorgungsforschung bspw. für die von der Weltgesundheitsorganisation (WHO) geforderte verbesserte „Stewardship“ im Gesundheitswesen: „Stewardship, sometimes more narrowly defined as governance, refers to the wide range of functions carried out by governments as they seek to achieve national health policy objectives. In addition to improving overall levels of population health, objectives are likely to be framed in terms of equity, coverage, access, quality, and patients' rights. National policy may also define the relative roles and responsibilities of the public, private and voluntary sectors - as well as civil society - in the provision and financing of health care" (www. who.int/healthsystems/stewardship/en). Dieser Bezug zu staatlichen Verantwortlichkeiten legt die Frage nahe, ob die Versorgungsforschung, welche ihren Schwerpunkt in der Beobachtung und Analyse der Strukturen und Prozesse in der klinischen Versorgung hat, auch für den Öffentlichen Gesundheitsdienst (ÖGD) mit seinen Schwerpunkten im Gesundheitsschutz, in der Prävention und in der Steuerung und Gestaltung im öffentlichen Raum Bedeutung hat. Bezogen auf den Öffentlichen Gesundheitsdienst als unmittelbar staatliche Institution im Dienst an der Bevölkerungsgesundheit ist zunächst eine Präzisierung wichtig: Ist eine Versorgungsforschung im ÖGD bzw. über den ÖGD gemeint, eine Versorgungsforschung durch den ÖGD, welche nur von wenigen Zentren mit entsprechenden Kompetenzen leistbar sein dürfte, oder eine Versorgungsforschung zur Nutzung für den ÖGD? Es ist diese letztere Möglichkeit, die Nutzung von Evidenz aus der Versorgungsforschung für den ÖGD, die zunächst im Vordergrund steht.

Mit Blick auf die oben genannten Aufgabengebiete des Öffentlichen Gesundheitsdienstes dürften insbesondere die Steuerungsaufgaben von einer aktiven Rezeption der Erkenntnisse der Versorgungsforschung Gewinn haben. Konkrete Ansatzpunkte bieten hierfür die Gesundheitsberichterstattung als traditionelles Aufgabengebiet des Öffentlichen Gesundheitsdienstes auf Bundes-, Landes- und kommunaler Ebene sowie regionale Steuerungskreise wie z.B. regionale Gesundheitskonferenzen oder psychosoziale Arbeitsgemeinschaften. Hier sei ein Blick nach vorne, eine neue Sicht auf alte Dinge, gewagt: In einem Gesundheitswesen der Zukunft, welches Gesundheitsschutz, Prävention und Gesundheitsförderung als Kontinuum in den Versorgungsalltag als Normalität integriert hat, wäre eine solche Unterteilung gegenstandslos. Ein Miteinander der Bereiche von Prävention, Gesundheitsförderung, Gesundheitsschutz, Versorgung und Rehabilitation wäre die Normalität.

Dies würde allerdings eine Entwicklung der Gesetzgebung und der untergesetzlichen Regularien voraussetzen, die im föderalen System Deutschlands nur schrittweise und in einem gemeinsamen Diskussionsprozess von Bund, Ländern und weiteren gesellschaftlichen Akteuren möglich ist. Es bedeutet perspektivisch auch eine weitergehende Integration, nämlich von Versorgungsforschung und Gesundheitssystemforschung. Dem ÖGD wie auch der Versorgungsforschung in Bund und Ländern sei auf diesem gemeinsamen Weg ein solches offenes und konstruktives aufeinander zu Gehen und ein gemeinsames Unterwegssein gewünscht, welches die hoffnungsvollen Ansätze einer New Public Health, von verantwortlicher Stewardship und einer „Gesundheit in allen Politikfeldern“ in der Praxis umsetzt. Dass auch solches Forschen und Handeln ein Dienst am Menschen ist, vergleichbar dem individualmedizinischen Handeln, sei noch einmal ausdrücklich bekräftigt. Die Weiterentwicklung der Versorgungsforschung ist eine wichtige Zukunftsaufgabe in Deutschland!

\section{Literatur}

1 Badura B, Busse R, Gostomzyk J et al. Memorandum zur Versorgungsforschung in Deutschland, Situation - Handlungsbedarf - Strategien. Dtsch Med Wochenschr 2004; 129: 771-773

2 Kohn LT, Corrigan JM, Donaldson MS, Hrsg. To Err Is Human - Building a Safer Health System. Committee on Quality of Health Care in America, Institute of Medicine. Washington DC: National Academy Press, 2000 URL http://www.nap.edu/openbook.php?isbn=0309068371 download 13.02.2015

3 Wennberg J, Gittelsohn A. Small area variations in health care delivery. Science 1973; 182: 1102-1108

4 McPherson $K$, Wennberg JE, Hovind $O B$ et al. Small-area variations in the use of common surgical procedures: an international comparison of New England, England, and Norway. N Engl J Med 1982; 307: $1310-1314$

5 Pflanz M. Die Epidemiologische Methode in der Medizinischen Soziologie. Kölner Zeitschrift für Soziologie und Sozialpsychologie 1958; 3: $134-149$ 